\title{
Analytical Evaluation of a Hybrid Chaotic Shift Keying [CS-QCSK] in a CDMA System
}

\author{
K. Thilagam \\ Research Scholar \\ Department of ECE \\ Pondicherry Engg.College
}

\author{
K. Jayanthi \\ Associate Professor \\ Department of ECE \\ Pondicherry Engg.College
}

\begin{abstract}
In recent years, various chaos based modulation schemes were evolved, of which the CS-DCSK modulation technique provides a better BER performance and bandwidth efficiency, due to its code domain approach[10]. The QCSK modulation technique provides double benefit, namely: higher data rate with similar BER performance and same bandwidth occupation as DCSK. By combining the advantage of code shifted differential chaos shift keying (CS-DCSK) and Quadrature chaos shift keying (QCSK) scheme, a novel CS-QCSK modulation scheme called code shifted Quadrature chaos shift keying is proposed. The noise performance of CS-QCSK is better to most conventional modulation schemes and also provides an increased data transmission rates with greatly improved robustness. Analytical expressions for the bit-error rates are derived for both AWGN channel and Rayleigh multipath fading channel. The simulation result shows that the proposed method outperforms the classical approaches in terms of bit error rate and spectral efficiency.
\end{abstract}

\section{Keywords}

Differential chaos shift keying (DCSK) ; Quadrature chaos shift keying (QCSK); Code shifted differential chaos shift keying (CS-DCSK); code shifted Quadrature chaos shift keying ( CS-QCSK); BER(Bit Error Rate)

\section{INTRODUCTION}

In the last few years, a great research effort has been devoted, concerning chaotic carrier modulation. In contrary to the conventional modulation schemes, a wideband, nonperiodic chaotic signal is used as carrier, which results in good correlation characteristics and robustness against multipath fading effects. The basic concept of chaos based modulation techniques for coherent and non-coherent systems have been analyzed and studied in [1]\&[2]. For a coherent system, the receiver needs an exact replica of the chaotic signal, such robust synchronization techniques are still not realizable in a practical environment [3]. It is worth mentioning the evolution of various types chaos based modulation schemes and their significance and this section is devoted for summarizing the literature survey/ research findings of such schemes. The DCSK scheme, discussed in [4] represents a more robust non-coherent scheme, in which the exact knowledge of the chaotic basis functions is not needed at the receiver. Multipath performance of binary
DCSK systems using a standard correlation detector was discussed in [5]. Quadrature CSK (QCSK) is a multilevel version of DCSK, based on the generation of an orthogonal chaotic functions. It allows an increase in data rate occupying same bandwidth with respect to DCSK [6]. In [7],[11] the performance of the DCSK system over a channel with Rayleigh fading or Rician fading was discussed with the necessity to model the effects of multipath delay spread as well as fading. A multiple-access technique with DCSK, using a one dimensional iterative map to generate the chaotic signals and similar average data rates for all the users have been discussed in [8]\&[12]. In [9] FM-QCSK discusses the generation of inherent wideband signal with constant energy per bit, which can be achieved by using the combination of frequency modulation with QCSK. The high-level constellation in FM-QACSK which can improve the speed of chaos shift keying is examined in [10] .The ultra wide band systems based on DCSK and FM-DCSK schemes and the implementation in both analog and digital technology is discussed in [13].

The DCSK system incorporated with two-user cooperative diversity technique and multiple accessing scheme is adopted and discussed in [14]. This had resulted in an excellent crosscorrelation characteristics of Walsh code sequences. CSDCSK uses code domain approach, therefore the reference and the information bearing signals are transmitted in the same time slots, it offers better BER performance and bandwidth efficiency, as discussed in [15]. In this paper, a novel CS-QCSK modulation scheme, which is the combination of CS-DCSK and QCSK modulation schemes, is proposed. The proposed scheme uses the code domain approach, where the reference and the information bearing signals are transmitted in the same time slots. This improved chaos based modulation scheme carries two bits in the information bearing signal part. Therefore, the transmission rate is maximized with the increased spectral efficiency and an improved BER performance can be achieved compared to the conventional modulation schemes. It also offers the advantages of both the CS-DCSK and QCSK schemes [15], [6]. The proposed work is basically a modified version of CSDCSK scheme, explored in [15]. The highlight of this proposal is basically to improve transmission data rates and spectral efficiency as compared to [15].Thus an improved chaos -based modulation scheme CS-QCSK has been 
recommended as an alternative to the work suggested in [15]. In this paper, a thorough analytical study for the proposed scheme is made to validate its supremacy.

The remaining part of the paper is organized as follows: In section 2, the overview of chaos based modulation schemes and the proposed system model is described. Section 3, elaborates the analytical BER derivation for different channel conditions. Simulation results are discussed in Section 4. Section 5, deals with the conclusion of the paper.

\section{OVERVIEW ON CHAOS BASED MODULATION SCHEMES}

In order to explain the CS-QCSK system, a brief introduction to DCSK, QCSK and CS- DCSK methods is essential and the details are given in this section.

\subsection{Differential Chaos Shift Keying (DCSK)}

In DCSK system, for each symbol period two chaotic sample functions are used to represent, one bit of information. The transmitted DCSK signal has two parts; the first part of the signal is reference signals, while the second denotes the information-bearing signals. If the bit ' 1 ' is to transmitted, then the information bearing signal is the replica of the reference part. If the bit ' 0 ' is to be transmitted, then the information bearing signal is the inverted copy of the reference part. The i-th symbol can be represented as,

$$
\mathrm{S}_{\mathrm{DCSK}}(\mathrm{t})=\left\{\begin{array}{cl}
\mathrm{x}(\mathrm{t}) & \mathrm{t}_{\mathrm{i}} \leq \mathrm{t}<\left(t_{i}+\frac{T}{2}\right) \\
\mp \mathrm{x}(\mathrm{t}-\mathrm{T} / 2) & \left(t_{i}+\frac{T}{2}\right) \leq t<\left(t_{i}+T\right)
\end{array}\right.
$$

Where, ' $\mathrm{T}$ ' is the symbol duration. In the receiver, the original information is extracted by computing the correlation between two received sample functions. The output of the correlator is sampled over each symbol period and the output is finally given to the decision device to determine the binary values 1 's or 0 's.

\subsection{Quadrature Chaos Shift Keying (QCSK)}

Similar to DCSK method, each symbol period of QCSK also has two parts, but the modification is, informationbearing signals holds two bits of information by means of quadrature chaos shift keying technique. Since two bits of information are transmitted, it is possible for QCSK scheme to obtain higher data rate. Quadrature chaotic signals are produced by an orthonormal basis chaotic sample functions. The transmitted QCSK signal is given by,

$$
\mathrm{S}_{\mathrm{QCSK}}(\mathrm{t})= \begin{cases}\sqrt{E_{b}} \mathrm{c}_{\mathrm{x}}(\mathrm{t}) & 0<\mathrm{t}<\frac{T}{2} \\ \sqrt{E_{b}}\left(\mathrm{a}_{1} \mathrm{c}_{\mathrm{x}}(\mathrm{t}-\mathrm{T} / 2)+\mathrm{a}_{2} \mathrm{c}_{\mathrm{y}}(\mathrm{t}-\mathrm{T} / 2)\right) & \frac{T}{2} \leq t<T\end{cases}
$$

Where, $\mathrm{E}_{\mathrm{b}}$ is the bit energy over $\mathrm{T} / 2$ period, satisfying the orthogonal relations. $\mathrm{C}_{\mathrm{X}}(\mathrm{t}-\mathrm{T} / 2)$ is chaotic signal with duration $\mathrm{T} / 2$ and $\mathrm{C}_{\mathrm{y}}(\mathrm{t}-\mathrm{T} / 2)$ is chaotic signal orthogonal to $\mathrm{C}_{\mathrm{X}}(\mathrm{t}-\mathrm{T} / 2)$ with duration $\mathrm{T} / 2$.

\subsection{Code Shifted - Differential Chaos Shift Keying (CS-DCSK)}

In DCSK, the reference and the information bearing signals are transmitted in two consecutive time slots because of its TDMA approach. This time domain approach provides two independent channels for the transmission of reference and information bearing signals. With this, it requires a delay component both in the modulator and demodulator circuits and halves the data rate. To overcome this drawback, an alternative approach used is CS-DCSK, where both the reference and the information bearing signals are transmitted in the same time slot because of its code domain approach (i.e.) the two signals are separated by Walsh codes instead of time delay. The transmitted CS-DCSK signal is given by,

$$
\mathrm{S}_{\mathrm{b}}(\mathrm{t})=\sum_{k=0}^{N-1} \mathrm{~W}_{\mathrm{R}, \mathrm{k}+1} \mathrm{C}(\mathrm{t}-\mathrm{kT} \mathrm{c})+\mathrm{a} \sum_{\mathrm{k}=0}^{\mathrm{N}-1} \mathrm{~W}_{\mathrm{I}, \mathrm{k}+1} \mathrm{C}\left(\mathrm{t}-\mathrm{kT}_{\mathrm{c}}\right) \quad \mathrm{T}_{\mathrm{S}}=\mathrm{NT}_{\mathrm{C}}
$$

Where, $\mathrm{W}_{\mathrm{R}, \mathrm{k}+1}$ is the reference signal, $\mathrm{W}_{\mathrm{I}, \mathrm{k}+1}$ is the information signal, $\mathrm{C}\left(\mathrm{t}-\mathrm{kT}_{\mathrm{C}}\right)$ is the chaotic signal, $\mathrm{T}_{\mathrm{S}}$ is the symbol duration and $\mathrm{T}_{\mathrm{C}}$ is the chip duration. The limitation of this system is, with the increased complexity the Bit Error Rate obtained by this system is more or less similar when compared with the existing system.

\subsection{System Model of CS-QCSK Modulation}

The aim of this section is to illustrate the practical importance of CS-QCSK modulation scheme, whose simplified transmitter and receiver block diagram are shown in Fig.1 and Fig.2 respectively. A baseband system is considered for simplicity. But it is understood, that if the scheme is to be employed for wireless communications a modulator is in need to generate the corresponding RF pass band signal. Furthermore, it is assumed that the description of the CS-QCSK scheme in the continuoustime domain admits an equivalent discrete-time representation. The discussion made in sections 2.2 and 2.3 vividly present the advantages of both QCSK and CS-DCSK schemes respectively. By combining these two techniques a novel CS-QCSK modulation scheme can be arrived at. In CS-QCSK, the symbol $\mathrm{S}$ is transmitted with the reference signal and information signal in the same time slot but separated by Walsh code sequences. The CS-QCSK transmitted signal is given by,

$$
\begin{aligned}
& \mathrm{S}_{\mathrm{b}}(\mathrm{t})=\sum_{k=0}^{N-1} \mathrm{~W}_{\mathrm{R}, \mathrm{k}+1} \mathrm{C}_{\mathrm{x}}\left(\mathrm{t}-\mathrm{kT} \mathrm{T}_{\mathrm{c}}\right)+\mathrm{a}_{1} \sum_{k=0}^{\mathrm{N}-1} \mathrm{~W}_{\mathrm{II}, \mathrm{k}+1} \mathrm{C}_{\mathrm{x}}\left(\mathrm{t}-\mathrm{kT} \mathrm{T}_{\mathrm{c}}\right)+\mathrm{a}_{2} \sum_{k=0}^{\mathrm{N}-\mathrm{I}} \mathrm{W}_{\mathrm{L} 2, \mathrm{k}+1} \mathrm{C}_{\mathrm{y}}\left(\mathrm{t}-\mathrm{kT} \mathrm{T}_{\mathrm{c}}\right) \quad \mathrm{T}_{\mathrm{S}}=\mathrm{NT}_{\mathrm{c}} \\
& \mathrm{C}_{\mathrm{x}}\left(\mathrm{t}-\mathrm{kT}_{\mathrm{c}}\right)=\left\{\begin{array}{cc}
\mathrm{c}_{\mathrm{x}}(\mathrm{t}) & \mathrm{kT}_{\mathrm{c}} \leq \mathrm{t}<(k+1) \mathrm{T}_{\mathrm{c}} \\
0 & \text { otherwise }
\end{array}\right.
\end{aligned}
$$

$$
\mathrm{C}_{\mathrm{y}}\left(\mathrm{t}-\mathrm{kT}_{\mathrm{c}}\right)=\left\{\begin{array}{cc}
\mathrm{c}_{\mathrm{y}}(\mathrm{t}) & \mathrm{kT}_{\mathrm{c}} \leq \mathrm{t}<(k+1) \mathrm{T}_{\mathrm{c}} \\
0 & \text { otherwise }
\end{array}\right.
$$

$$
\Delta=\int_{0}^{T_{s=N T}} \sum_{k=0}^{N-1} \mathrm{~W}_{\mathrm{R}, \mathrm{k}+1} \mathrm{C}_{\mathrm{x}}\left(\mathrm{t}-\mathrm{kT} \mathrm{T}_{\mathrm{c}}\right) \mathrm{a}_{1} \sum_{k=0}^{\mathrm{N}-1} \mathrm{~W}_{\mathrm{II}, \mathrm{k}+1} \mathrm{C}_{\mathrm{x}}\left(\mathrm{t}-\mathrm{kT} \mathrm{T}_{\mathrm{c}}\right) \mathrm{a}_{2} \sum_{k=0}^{\mathrm{N}-1} \mathrm{~W}_{\mathrm{I} 2, \mathrm{k}+1} \mathrm{C}_{\mathrm{y}}\left(\mathrm{t}-\mathrm{kT} \mathrm{T}_{\mathrm{c}}\right) \cdot d t
$$

Where ' $\Delta$ ' is the inner product term. 


$$
\begin{aligned}
& E_{b}=\int_{0}^{T_{s}} c^{2}(t) c_{y}(t) \cdot d t \\
& (.)^{T}=\text { Transporse of vector. } \\
& \mathrm{c}_{\mathrm{x}}(\mathrm{t})=\mathrm{C}_{\mathrm{x}}\left(\mathrm{t}-\mathrm{kT}_{\mathrm{c}}\right) ; \forall k \\
& \mathrm{c}_{y}(\mathrm{t})=\mathrm{C}_{y}\left(\mathrm{t}-\mathrm{kT}_{\mathrm{c}}\right) ; \forall k
\end{aligned}
$$

The orthogonality of the signal is assured by Walsh code sequences; therefore the reference and the information signal are independent of the chaotic carrier.

Referring to the Fig.1 transmitter block diagram, the CSQCSK modulation scheme consist of chaos signal generator, which generates chaos signal $\mathrm{C}_{\mathrm{X}}(\mathrm{t})$ and its orthogonal signal $\mathrm{C}_{\mathrm{y}}(\mathrm{t})$. The orthogonal signal is obtained by taking the Hilbert transform of the chaos signal $\mathrm{C}_{\mathrm{X}}(\mathrm{t})$. The transmitter block consists of three Walsh function generator, which generates three different Walsh code sequences that are orthogonal in nature. It has $(\mathrm{N}-1)$ delay elements of $\mathrm{T}_{\mathrm{c}}$ chip duration. The three Walsh function generator, generate Walsh codes, $\mathrm{W}_{\mathrm{R}, \mathrm{k}+1}$ for reference signal, and $\mathrm{W}_{\mathrm{I} 1, \mathrm{k}+1}$ , W $2, \mathrm{k}+1$ for information signal. The reference signal of Walsh code $\mathrm{W}_{\mathrm{R}, \mathrm{k}+1}$ is multiplied with the chaos signal and the corresponding output is obtained by the switching unit S1. It has a bit splitter, which splits the input bit stream into odd and even sequences. The information signal of Walsh

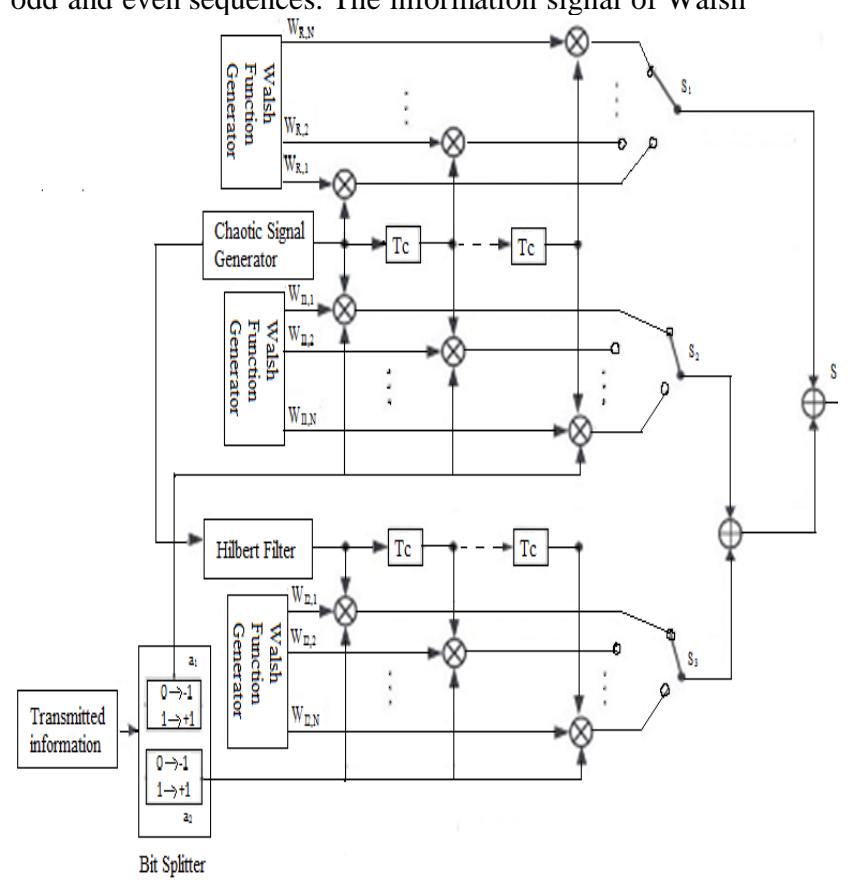

Fig.1 Block diagram of CS-QCSK Transmitter

code $\mathrm{W}_{\mathrm{I} 1, \mathrm{k}+1}$ is multiplied with the odd sequence of bits and the chaos signal, the corresponding output is obtained by the switching unit $\mathrm{S}_{2}$. The information signal of Walsh code WI2,k+1 is multiplied with the even sequence of bits and the orthogonal chaos signal, the corresponding output is obtained by the switching unit $\mathrm{S}_{3}$.The output obtained at the switching unit $\mathrm{S}_{2}$ and $\mathrm{S}_{3}$ are summed and it is further summed with the switching unit S1 to obtain the final output. The CS- QCSK signal thus is finally obtained.

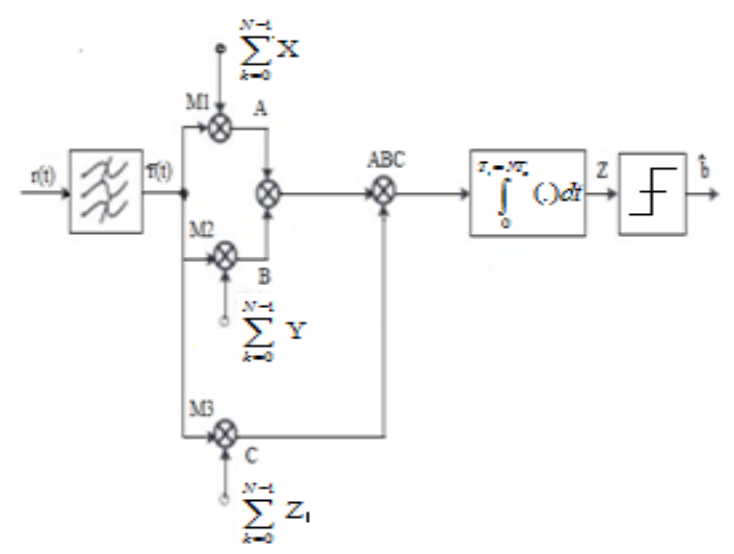

Fig.2 Block diagram of CS-QCSK Receiver

The above Fig.2 shows the block diagram of CS-QCSK receiver. The receiver unit consists of band pass filter with the bandwidth of $2 \mathrm{~B}$ which can pass the received signal $\mathrm{r}(\mathrm{t})$ without any distortion. The receiver filter output with Additive White Gaussian Noise (AWGN) n (t) is obtained as,

${ }_{r}(t)=\sum_{k=0}^{N-1} \mathrm{~W}_{\mathrm{R}, \mathrm{k}+1} \mathrm{C}_{\mathrm{x}}\left(\mathrm{t}-\mathrm{kT} \mathrm{T}_{\mathrm{c}}\right)+\mathrm{a}_{1} \sum_{k=0}^{\mathrm{N}-1} \mathrm{~W}_{\mathrm{Il}, \mathrm{k}+1} \mathrm{C}_{\mathrm{x}}\left(\mathrm{t}-\mathrm{kT} \mathrm{T}_{\mathrm{c}}\right)+\mathrm{a}_{2} \sum_{k=0}^{\mathrm{N}-1} \mathrm{~W}_{\mathrm{I}, \mathrm{k}+\mathrm{k}+1} \mathrm{C}_{\mathrm{y}}\left(\mathrm{t}-\mathrm{kT} \mathrm{T}_{\mathrm{c}}\right)+\mathrm{n}(\mathrm{t})$

$$
\operatorname{rect}_{\mathrm{x}}\left(\mathrm{t}-\mathrm{kT}_{\mathrm{c}}\right)=\left\{\begin{array}{lr}
1 & , \mathrm{kT}_{\mathrm{c}} \leq \mathrm{t}<(k+1) \mathrm{T}_{\mathrm{c}} \\
0 & , \text { otherwise }
\end{array}\right.
$$

$$
\operatorname{rect}_{\mathrm{y}}\left(\mathrm{t}-\mathrm{kT}_{\mathrm{c}}\right)=\left\{\begin{array}{rc}
1 & , \mathrm{kT}_{\mathrm{c}} \leq \mathrm{t}<(k+1) \mathrm{T}_{\mathrm{c}} \\
0 & , \text { otherwise }
\end{array}\right.
$$

The receiver filter output is further given to the three mixer unit M1, M2 and M3.In the mixer unit, the filter output signal is multiplied by a rectangular function weighted by the three Walsh code sequences. The transmitted signal is of Walsh codes incorporated with three terms. In order to obtain those terms $\mathrm{A}, \mathrm{B}$ and $\mathrm{C}$ the receiver filter output is multiplied by a rectangular function. The output of M1, M2 and M3 is multiplied to obtain the signal ABC. The multiplier output is then given to the integrator unit to obtain the observation vector ' $Z$ ' which is then fed to the hard decision device, where the bits are estimated by the decision rule. Using Walsh code, the transmitted signal is incorporated with three terms A, B and $\mathrm{C}$. The three terms $\mathrm{A}, \mathrm{B}$ and $\mathrm{C}$ can be obtained by multiplying equation (4) that corresponds to reference signal and information signals respectively. The terms $\mathrm{X}, \mathrm{Y}$ and $\mathrm{Z}$ in the receiver block are 


$$
\begin{aligned}
& \mathrm{X}=\mathrm{W}_{R, \mathrm{k}+1} \operatorname{rect}_{\mathrm{x}}\left(\mathrm{t}-\mathrm{kT}_{\mathrm{c}}\right), \mathrm{Y}=\mathrm{W}_{\mathrm{I} 1, \mathrm{k}+1} \operatorname{rect}_{\mathrm{x}}\left(\mathrm{t}-\mathrm{kT}_{\mathrm{c}}\right), \\
& \mathrm{Z} 1=\mathrm{W}_{\mathrm{I} 2, \mathrm{k}+1} \operatorname{rect}_{y}\left(\mathrm{t}-\mathrm{kT}_{\mathrm{c}}\right)
\end{aligned}
$$

\section{ANALYTICAL APPROACH UNDER VARYING CHANNELS}

The analytical expression for BER of CS-QCSK over AWGN and the multipath Rayleigh fading channels are derived in the following section

\subsection{Additive White Gaussian Noise (AWGN) Channel}

The observation vector ' $Z$ ' obtained at the integrator output stage in the presence of AWGN noise is given by,

$$
\mathrm{Z}=\sum_{k=0}^{N-1} \int_{\mathrm{kT}_{\mathrm{c}}}^{(\mathrm{k}+1) \mathrm{T}_{\mathrm{c}}}\left[\mathrm{W}_{\mathrm{R}, \mathrm{k}+1} \cdot{ }_{r}(t)\right]\left[\mathrm{W}_{\mathrm{I} 1, \mathrm{k}+1} \mathrm{~W}_{\mathrm{I} 2, \mathrm{k}+1} \cdot{ }_{r}(t)\right] \mathrm{dt}
$$

$$
=\sum_{\mathrm{k}=0}^{\mathrm{N}-1} \mathrm{~W}_{\mathrm{R}, \mathrm{k}+1} \mathrm{~W}_{\mathrm{I} 1, k+1} \mathrm{~W}_{\mathrm{I} 2, \mathrm{k}+1} \int_{\mathrm{kT}}^{(\mathrm{k}+1) \mathrm{T}_{\mathrm{c}}}\left[\left(\mathrm{W}_{\mathrm{R}, \mathrm{k}+1}+\mathrm{a}_{1} \mathrm{~W}_{\mathrm{II}, \mathrm{k}+1}+\mathrm{a}_{2} \mathrm{~W}_{\mathrm{I} 2, k+1}\right)+c\left(t-k T_{c}\right)+n(t)\right]^{2} \cdot d t
$$

There are different types of chaotic maps to generate the chaotic signals. In the proposed method, logistic chaotic map is considered and is defined as, $x(n+1)=1-2 x^{2}(n)$. Where, $\beta=\mathrm{T}_{\mathrm{C}} \mathrm{f}_{\mathrm{S}}$ is the number of samples in a chip time ' $\mathrm{T}_{\mathrm{c}}$ ' and $\mathrm{K}=\mathrm{T}_{\mathrm{S}} \mathrm{f}_{\mathrm{S}}=\mathrm{N} \beta$ where ' $\mathrm{T}_{\mathrm{s}}$ ' is the symbol duration and ' $\mathrm{K}$ ' is referred as the spreading factor. Consider, the transmission of $\mathrm{a} 1=\mathrm{a} 2=+1$ sequence. Since, the logistic map is defined in the continuous time domain it is essential to convert it into the discrete time domain. Consider $\mathrm{C}_{\mathrm{j}}$ be the samples of chaotic signal and $\eta_{j}$ be the sampled channel noise. Then, the equation for discrete time equivalent is given as,

$$
Z=\sum_{\mathrm{k}=0}^{\mathrm{N}-1} \mathrm{~W}_{\mathrm{R}, \mathrm{k}+1} \mathrm{~W}_{\mathrm{Il}, \mathrm{k}+1} \mathrm{~W}_{\mathrm{I} 2, \mathrm{k}+1} \sum_{\mathrm{m}=1}^{\beta}\left[\left(\mathrm{W}_{\mathrm{R}, \mathrm{k}+1}+\mathrm{a}_{1} \mathrm{~W}_{\mathrm{Il}, \mathrm{k}+1}+\mathrm{a}_{2} \mathrm{~W}_{\mathrm{I} 2, \mathrm{k}+1}\right) \cdot c_{k \beta+m}+\eta_{k \beta+m}\right]^{2}
$$

Where,

$$
c_{k \beta+m}=\left\{\begin{array}{lr}
c_{m} & , \kappa \beta \leq \kappa \beta+m<(k+1) \beta \\
0 & \text {, otherwise }
\end{array}\right.
$$

From the equation (11), the decision variable is decomposed into three terms,

$$
Z=Z_{A}+Z_{B}+Z_{C}
$$

For logistic maps, the expectation of chaos signal is $E\left\{C_{j}^{2}\right\}=1 / 2$ and variance of chaos signal is
$\operatorname{Var}\left\{C_{j}^{2}\right\}=1 / 8$. It is assumed, that the probability distribution of the observation variable can be approximated by Gaussian approximation and the transmitted bits are equiprobable. Therefore, after simplification the bit error rate of CS-QCSK modulation scheme is obtained as,

$$
B E R_{C S-Q C S K}=\frac{1}{2} \operatorname{erfc}\left(\frac{3\left(E_{b} / N_{0}\right)}{\sqrt{\frac{3}{4} K+\frac{1}{2} K+2\left(E_{b} / N_{0}\right)}}\right)
$$

\subsection{Rayleigh Multipath Fading Channel}

Considering the Rayleigh multipath fading channels, the received signal is denoted as,

$$
r(t)=h(t) \otimes x(t)+n(t)
$$

and the channel impulse response is given as

$$
h(t)=\sum_{l=0}^{L-1} \alpha_{l} \delta(l-\eta)
$$

Where, $\mathrm{L}$ is the number of propagation paths, $\alpha_{1}$ gain of the lth path, $\tau$ l is the delay of the $1^{\text {th }}$ path and $\delta(t)$ denotes the dirac delta function. The gains $\alpha_{1}$ of the propagation paths are assumed to be independent identical distributed Rayleigh random variables. The BER measured in Rayleigh multipath channel is an approximation developed from the noise performance of AWGN channel. The conditional BER measured for the multipath is given by,

$\operatorname{BER}\left(\alpha_{0}, \alpha_{1} \ldots \ldots \ldots \alpha_{L-1}\right)=\operatorname{BER}\left(\gamma_{b}\right)$

Where, $\gamma_{b}=\left(E_{b} / N_{O}\right)\left(\alpha_{0}, \alpha_{1}\right.$ $\left.\alpha_{L-1}\right)=\left(\gamma_{0}+\gamma_{1}\right.$

$\left.+\ldots \ldots \ldots \ldots \ldots \ldots+\gamma_{L-1}\right)$

In this paper, it is assumed to have multipath channels with three propagation paths, then the error rate expressions are,

$$
B E R_{C S-Q C S K}=\frac{1}{2} \operatorname{erfc}\left(\frac{3\left(\gamma_{b}\right)}{\sqrt{\frac{3}{4} K+\frac{1}{2} K+2\left(\gamma_{b}\right)}}\right)
$$

In order to validate the analytical approach of the proposed scheme, an attempt is made through simulations. The next section presents the simulation analysis of the proposed scheme and it is finally compared with the analytical results.

\section{SIMULATION ANALYSIS}

The BER performances of CS-QCSK modulation schemes for different channel conditions are also analyzed through Matlab simulation. 
Table 1. Simulation Parameters for the Analysis

\begin{tabular}{|c|c|}
\hline Parameters & Values \\
\hline Chip duration(Tc) & 2millisecond \\
\hline Symbol duration(Ts) & 2 micro second \\
\hline Transmission power(P) & $43 \mathrm{dBm}$ \\
\hline $\begin{array}{c}\text { Noise power spectral } \\
\text { density(No) }\end{array}$ & 1 \\
\hline Spread factor(K) & $4,8,20$ \\
\hline Channel types & $\begin{array}{c}\text { AWGN and } \\
\text { Rayleigh }\end{array}$ \\
\hline
\end{tabular}

Initially, the BER performance of AWGN channel is discussed, followed by multipath Rayleigh fading channel.

\subsection{BER Performance over AWGN Channel}

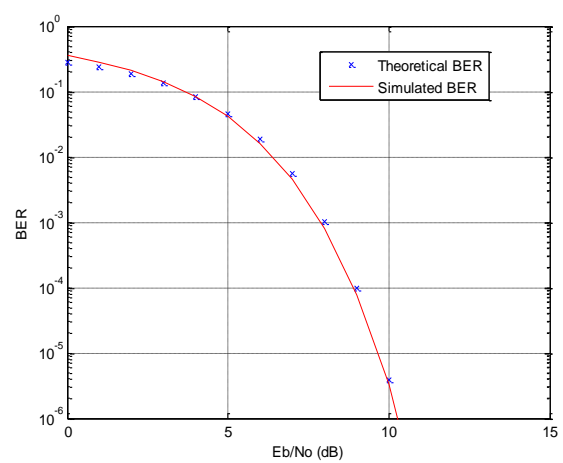

Fig. 3. BER performance of CS-QCSK modulation scheme for AWGN channel.

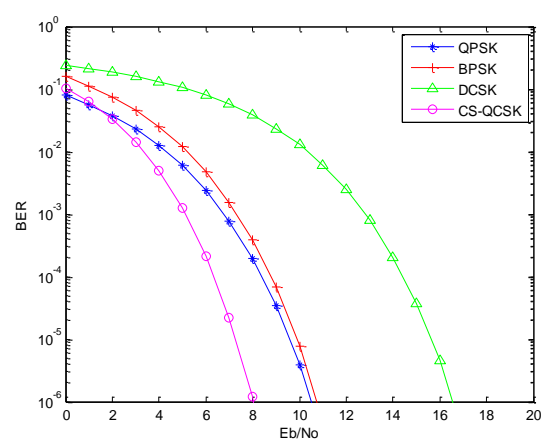

Fig. 4. BER performance comparison for different modulation schemes with CS-QCSK modulation scheme Over AWGN channel.

From the fig.3, it is inferred that, BER performance of CSQCSK scheme is plotted with the obtained theoretical and simulated values. The BER values calculated from the analytical expression and the simulation are very similar and both the graphs merge with each other. For the BER value of $10^{-6}$, the required $(\mathrm{Eb} / \mathrm{No})$ value is $10 \mathrm{~dB}$ (approximately).From fig. 4 it is observed that the proposed schemes outperforms the other conventional schemes with the improved BER performance under AWGN channel.

\subsection{BER Performance over Rayleigh Multipath Channel}

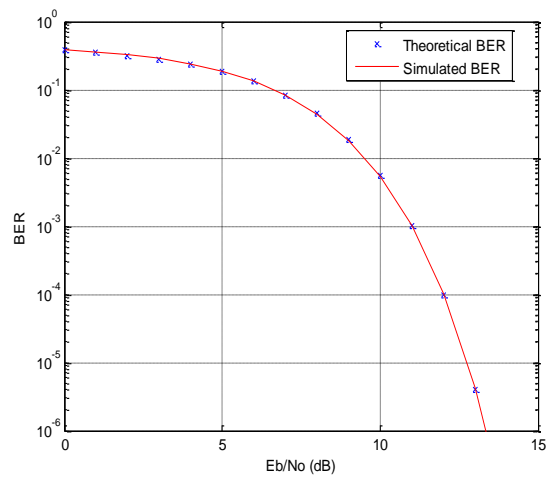

Fig. 5. BER performance of CS-QCSK modulation scheme for Rayleigh multipath channel.

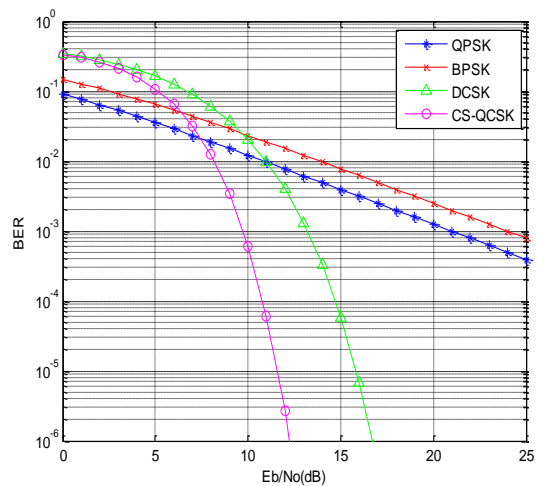

Fig. 6. BER performance comparison for different modulation schemes with CS-QCSK modulation scheme Over AWGN channel.

From the above figure, it can be observed that BER performance of CS-QCSK scheme is sketched out for both obtained theoretical and simulated values. For the BER value of $10^{-6}$, the required (Eb/No) value is $11.1 \mathrm{~dB}$ (approximately). .From fig. 6 it is observed that the proposed schemes outperforms the other conventional schemes with the improved BER performance under Rayleigh channel.

\subsection{Spectral Efficiency Comparison for Various Modulation schemes}

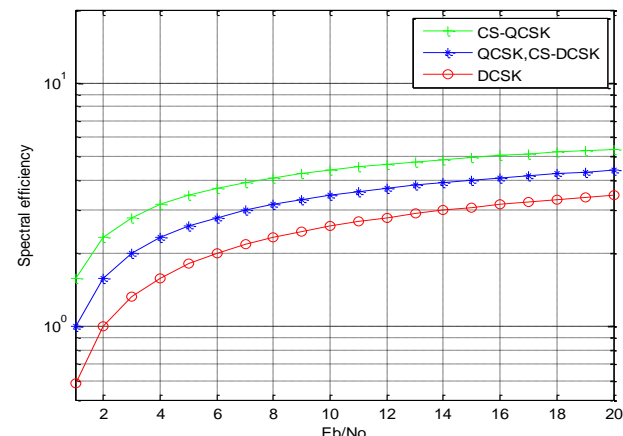

Fig. 7. Spectral efficiency performance comparison for different modulation schemes with CS-QCSK modulation scheme. 
In general, the region under the spectral curve provides a reliable communication. From the fig.7, it is inferred that the proposed scheme CS-QCSK achieves better spectral efficiency compared to the other conventional schemes. It means that the region under the curve of the proposed system is vast compared to the other schemes.

\section{Table 2.Comparison of Eb/No for the different channel} conditions with various spreading factors for CS-QCSK scheme

\begin{tabular}{|c|c|c|c|c|}
\hline Parameter & $\begin{array}{c}\text { Channel } \\
\text { Type }\end{array}$ & K=4 & K=8 & K=20 \\
\hline Eb/No(dB) & AWGN & 8 & 8.6 & 8.9 \\
\hline Eb/No(dB) & Rayleigh & 11 & 11.7 & 12 \\
\hline
\end{tabular}

' $\mathrm{K}$ ' is the Spread factor, BER $=10^{-6}$. Table 2. Gives the comparison of the $\mathrm{Eb} / \mathrm{No}$ values for various spread factors under different channel conditions. In general as the spread factor increases it requires maximum bandwidth; therefore it requires maximum $\mathrm{Eb} / \mathrm{No}$ value. But, it is inferred that the proposed (CS-QCSK) scheme achieves better BER performance with minimum spread factor thereby requiring minimum $\mathrm{Eb} / \mathrm{No}$ value.

\section{CONCLUSION}

In the field of chaos-based communication, M-ary DCSK scheme seems to be the best known modulation scheme. But, the drawback is, it requires $(\mathrm{N}-1)$ number of delay lines both in the transmitter and the receiver units. In this paper, an improved chaos-based communication method using CSQCSK technique is recommended as an alternative to [10]. The proposed CS-QCSK scheme, avoids the use of delay lines in the receiver units. It transmits the reference and information signals in the same time slot and offers an increase in data rate, better BER performance and bandwidth efficiency. The performance of the proposed modulation scheme is analyzed under both AWGN and multipath channels. Analytical expressions for BER of CSQCSK are derived and verified with matlab simulation. Simulation results, shows that the CS-QCSK exhibits better BER performance in AWGN and Rayleigh fading channels. This proves the suitability of the proposed modulation technique for the forthcoming mobile standards, which is the need of the hour.

\section{REFERENCES}

[1] A. Abel and W. Schwarz, "Chaos communicationsPrinciples, schemes, and system analysis," Proc. IEEE, Vol. 90, pp. 691-710, May 2002.

[2] G. Kolumbán, M. P. Kennedy, Z. Jako, and G. Kis, "Chaotic communications with correlator receivers: Theory and performance limits," Proc.IEEE, vol. 90, pp. 711-732, May 2002.
[3] H. Dedieu, M. P. Kennedy, and M. Hasler, "Chaos shift keying: Modulation and demodulation of a chaotic carrier using self-synchronizing Chua's circuits," IEEE Trans. Circuits Syst. II, vol. 40, pp. 634-642, Oct.1993.

[4] G. Kolumbán, B. Vizvári, W. Schwarz, and A. Abel, "Differential chaos shift keying: A robust coding for chaos communication," Proc. Int.Workshop Nonlinear Dynamics of Electronic Systems, pp. 87-92, June 1996.

[5] M. P. Kennedy, G. Kolumbán, G. Kis, and Z. Jákó, "Performance evaluation of FM-DCSK modulation in multipath environments," IEEE Trans.Circuits Syst. I, Fundam. Theory Appl., vol. 47, no. 12, pp. 1702-1711, Dec.2000.

[6] Z. Galias and G. M. Maggio, "Quadrature chaos-shift keying: Theory and performance a $\mathrm{n}$ a $\mathrm{ly} \mathrm{s}$ i s ," IEEE Trans. Circuits Syst. , Fundam.Theory Appl., vol. 48, no. 12, pp. 1510- 1519, Dec. 2001.

[7] S. Mandal and S. Banerjee, "Performance of differential chaos shift keying over multipath fading channels," in Proc. Indian Nat. Conf. Nonlinear Systems and Dynamics, Kharagpur, India, Dec. 2003.

[8] G. Kolumbán, P. Kennedy, and G. Kis, "Multilevel differential Chaos shift keying," in Proc. Int. Workshop, Nonlinear Dynamics of Electronics Systems, NDES'97, Moscow, Russia, 1997, pp. 191-196.

[9] YiWei Zhang, Xubang Shen and Yi Ding, "Design and performance analysis of an FM- QCSK chaotic communication system," International Conference on Wireless Communications, Networking and Mobile Computing, China, pp: 1-4, Sept. 2006.

[10] Jiamin Pan, He Zhang, "Design of FM-QACSK Chaotic Communication System" IEEE Trans.Wireless Communications \& Signal Processing, 2009.

[11] Xia, Y.,Tse,C.K.\&Lau, F.C.M, "Performance of Differential chaos shift keying digital communication systems over a multipath fading channel with delay spread,'IEEE Trans. Circuits Syst.-II 51,680-684,2004.

[12] Yao, J. \& Lawrance, A.J, "Performance analysis and optimization of multi-user differential chaos-shift keying communication systems," IEEE Trans.circ. Syst.-I 53, 2075-2091, 2006.

[13] Wang, L., Zhang, C. \& Chen, G. "Performance of an SIMO FM-DCSK communication system," IEEE Trans.Circuits Syst.-II 55, 457-461, 2008.

[14] Min, X., Xu, W., Wang, L. \& Chen, G., "Promising performance of an FM-DCSK UWB system under indoor environments," J.ET Commun. 4, 125-134, 2010.

[15] G.Koluban, W.K.Xu and L.Wang, "A Novel differential chaos shift keying modulation scheme," International journal of Bifurcation and chaos, 2011, Vol.21, No.3, 799-814. 